\title{
Surface Coating and functionalization of Metallic Biomaterials with Essential Oils for Antibacterial Applications
}

\author{
Martina Cazzola 1,*, Sara Ferraris ${ }^{1}$, Valeria Allizond ${ }^{2}$, Giuliana Banche ${ }^{2}$, \\ Cinzia Margherita Bertea 3, Giovanna Gautier di Confiengo ${ }^{4}$, Chiara Novara 1, \\ Andrea Cochis ${ }^{5,6}$, Lia Rimondini ${ }^{5,6}$ and Silvia Spriano ${ }^{1}$ \\ 1 Department of Applied Science and Technology, Politecnico di Torino, C.so Duca degli Abruzzi 24, \\ 10129 Torino, Italy; sara.ferrraris@polito.it (S.F.); chiara-novara@polito.it (C.N.); \\ silvia.spriano@polito.it (S.S.) \\ 2 Department of Public Health and Paediatrics, Microbiology Division, University of Turin, via Santena 9, \\ 10126 Turin, Italy; valeria.allizond@unito.it (V.A.); giuliana.banche@unito.it (G.B.) \\ 3 Plant Physiology Unit, Department of Life Sciences and Systems Biology, University of Turin, \\ Via Quarello 15/A, 10135 Turin, Italy; cinzia.bertea@unito.it \\ 4 CNR, IMAMOTER, Strada delle Cacce 73, 10135 Torino, Italy; g.gautier@imamoter.cnr.it \\ 5 Università del Piemonte Orientale UPO, Department of HealthSciences, Via Solaroli 17, 28100 Novara, \\ Italy; andrea.cochis@med.uniupo.it (A.C.); lia.rimondini@med.uniupo.it (L.R.) \\ 6 Center for Translational Research on Autoimmune \& Allergic Diseases - CAAD, Cso Trieste 15/A, \\ 28100 Novara, Italy \\ * Correspondence: martina.cazzola@polito.it
}

Received: 15 February 2019; Accepted: 5 March 2019; Published: 7 March 2019

\begin{abstract}
Essential oils (EO) are complex mixtures of natural molecules with a great activity against bacteria, virus and fungi. Despite their wide use in folk medicine, their employment in medicine or in biomedical products is still scarce and there are only few studies in literature. Their antibacterial and anti-adhesive properties are of interest for the development of coatings or functionalization of implant surfaces, but also for non-implantable medical devices, with the advantage of a reduced bacterial contamination, without the risk of developing bacteria resistance. For a first attempt, a peppermint essential oil was used in this research. The aim of the work was to functionalize or to coat the surface of different materials (Ti6Al4V, Steel 316L) with different surface finishing (grinded, polished, and chemically-treated). The obtained samples were characterized by means of XPS, FTIR, Fluorescent microscope observations and tape test. These analyses highlighted the presence of grafted oil biomolecules, or of a coating, on all the substrates after the procedure of functionalization/coating with remarkable chemical stability (during soaking in water or washing with acid or basic solutions) and mechanical adhesion. The samples were also subjected to antibacterial tests selected in order to simulate non-implantable applications: the test showed a reduction of the adherent bacteria and their viability on coated surfaces. In conclusion, the research work shows the great potential of the use of essential oils for surface functionalization or coating of biomaterials to obtain enhanced antibacterial properties: according to the final application, different oils can be selected in order to obtain the desired effects.
\end{abstract}

Keywords: antibacterial; essential oil; coating; metal alloys 


\section{Introduction}

As defined by European Pharmacopoeia [1], the essential oils are "Odorant product, generally of a complex composition, obtained from a botanically defined plant raw material, either by driving by steam of water, either by dry distillation or by a suitable mechanical method without heating. An essential oil is usually separated from the aqueous phase by a physical method that does not lead to significant change inits chemical composition".

Essential oils (EO) have attracted great interest in recent years within the scientific community. They have been used in the folk medicine since ancient time and they are recently used in the production of food packaging and medical devices. Essential oils have well known antibacterial activity, being interesting for multi-purpose uses because they could allow to avoid the side effects of antibiotics. In addition to antibacterial properties, they have also a wide variety of effects on human health such as cardiovascular protective effects, antitumoral effects, antinflammatory effects and many other positive impacts in the diseases involving free radical proliferations because of their antioxidant properties [2].

The EO have shown effects both on gram-positive and gram-negative bacteria [3] and they can be one of the responses to the increasing request of novel antibacterial agents. They are able to disrupt the bacterial wall interfering with the cell energy balance and damaging the cytoplasm [4]. The essential oils are also able to reduce the resistance of bacteria to antibiotics [5]. In the case of EO extracted from Mentha Species, the antibacterial activity is attributable in particular to the presence of alkaloids and phenols [6]. Menthapiperita (peppermint) essential oils which have a great amount of menthol and menthone have antibacterialactivity, synergic antibacterial activity with antibiotics and the ability to increase cell permeability [7].

Despite the increasing interest on application of the essential oils coupled with biomaterials, in literature there are no papers dealing with the direct coating or functionalization of metallic surfaces with EO. The aim of the work is to coat stainless steel 326L and Ti6Al4V alloy for antibacterial purposes in non-implantable applications in biomedical fields and to functionalize the surface of a chemically-treated bioactive Ti alloy with EO biomolecules for implantable applications.

\section{Materials and methods}

\subsection{Preparation of Ti6Al4V and Steel 316L Samples}

For the work, disks of Ti6Al4V Gr5 $2 \mathrm{~mm}$ thick, $10 \mathrm{~mm}$ in diameter, and disks of steel 316L $2 \mathrm{~mm}$ thick and $14 \mathrm{~mm}$ in diameter were used.

The samples were polished with an abrasive SiC paper up to 2500 grit ad than washed in order to clean them. Some of the Ti6Al4V samples were chemically treated in order to make the surface bioactive and rich in hydroxyl groups. The treatment was performed as explained in [8-10]. The treated samples were named "Chemically-treated" (CT).

\subsection{Functionalization/Coating with Mentha Piperita Essential oil}

For coating/functionalization, Mentha piperita essential oil (EssentialMenta, Pancalieri, Italy) was used.

For the coating procedure, the samples were put in a plate, covered with $5 \mu \mathrm{L}$ of EO and incubated in the dark at $37^{\circ} \mathrm{C}$ for $48 \mathrm{~h}$. After the polymerization step, the samples were washed in water and dried at room temperature. The coating was obtained on the samples of Ti6Al4V and steel 316L polished up to 2500 grit and on some samples of steel 316L polished up to 400 grit. The name of the samples was respectively Ti_Mint, steel_mint and steel 400_mint.

The functionalization procedure was performed by soaking the samples in a solution of $50 \%$ $\mathrm{EO} / 50 \%$ ethanol for $48 \mathrm{~h}$ at $37^{\circ} \mathrm{C}$, then the samples were washed a first time in ethanol, a second time in water and dried at room temperature. The samples obtained were named CT_50\% mint (funz). 


\subsection{Fluorescent Microscope Observations}

Presence of the EO coating on the surface of the Ti6Al4V and Steel samples was observed with a fluorescence microscope (Leica DM5500 BLeica Microsystems, IL, USA) exploiting the autofluorescence properties of the essential oil [11]. The analysis was also performed after $3 \mathrm{~h}, 7 \mathrm{~h}, 3$ days and 7 days of soaking in water to check the stability of the coating in contact with water.

\subsection{Tape Test}

The tape test was performed in accordance with the standard ASTM D 335L [12]. It was used to evaluate the coating adhesion to the steel 316L surface. The test was performed on Steel samples and evaluated by optical microscope observations.

\subsection{Resistance to Wash}

In order to test the resistance of the coating to typical cleaning solutions, the samples were cleaned with a wet sponge using three different solutions. An acid solution of $\mathrm{H}_{2} \mathrm{SO}_{4} 0.1 \mathrm{~N}$, a basic solution of $\mathrm{NaOH} 0.1 \mathrm{~N}$ and a commercial degreaser (Vegetale, Nuncas, Milan, Italy) [13,14]. After washing, the presence of the coating was evaluated macroscopically and by FTIR measurement (FTIR Hyperion 2000-Tensor 27, Bruker Optics, Ettlingen, Germany).

\subsection{XPS Analysis}

X-ray Photoelectron Spectroscopy analysis (XPS, PHI 5000 VERSAPROBE, PHYSICAL ELECTRONICS, Chanhassen, MN, USA) was performed on the surface of CT and CT_50\% mint (funz) in order to show the chemical groups exposed on the surfaces and to check the success of the functionalization procedure.

\subsection{Antibacterial Test}

An antibacterial test simulating the condition of contamination of a surface for non-implantable applications with Escherichia coli was performed.

Strain

A commercially available Escherichia coli strain (E. coli, ATCC 25922, from the American Type Culture Collection, Manassas, VA, USA) was used for the experiments. Bacteria were cultured following Manufacturer's instructions. Briefly, frozen samples were rinsed with 5-6 $\mathrm{mL}$ of fresh Luria Bertani (LB, Sigma, Milan, Italy) for $24 \mathrm{~h}$ at $37^{\circ} \mathrm{C}$. Then, few drops $(50 \mu \mathrm{L})$ were spotted into an LB agar plate and incubated $24 \mathrm{~h}$ at $37^{\circ} \mathrm{C}$ until single round colonies were formed. The plate was stored at $4{ }^{\circ} \mathrm{C}$ until use.

\section{Samples Infection}

Sterile samples were singularly seeded into $15 \mathrm{~mL}$ tubes by sterile tweezers. Sterile ultrapure water (Millipore) was contaminated by dispersing 3-4 bacteria colonies collected from the LB agar plate; the solution was adjusted to a $1 \times 10^{5}$ cells $/ \mathrm{ml}$ concentration by optical density (o.d., corresponding to a 0.001 o.d.@600 nm) using a spectrophotometer (Victor, Packard Bell, LA, USA) and then further diluted until the final concentration was $1 \times 10^{3}$ cells $/ \mathrm{ml}$. Afterwards, the samples stored into the $15 \mathrm{~mL}$ tubes were submerged with $5 \mathrm{~mL}$ of the contaminated water and incubated at $37^{\circ} \mathrm{C}$ under continuous shaking $(120 \mathrm{rpm})$ to simulate a flux of running water.

\section{Antibacterial Activity}

After 24 and $48 \mathrm{~h}$ of direct contact with the running infected water, the surface of the test samples was analyzed by means of adhered colonies viability and number using the alamar blue and CFU count assays, respectively. 
For the metabolic evaluation, the specimens were collected from the tubes, washed gently 3 times with PBS and moved to a new 24 multiwell plate; then, $1 \mathrm{~mL}$ of the ready-to-use alamar blue solution (alamarBlue ${ }^{\circledR}$ from Life Technologies, Milan, Italy) was added to each sample and the plate was incubated at the dark for $3 \mathrm{hrs}$.

Then, $100 \mathrm{ml}$ were collected from each well, spotted into a black-bottom 96 wells plate and the fluorescent signal evaluated by a spectrophotometer (Victor) at $490 \mathrm{~nm}$ wavelength.

Statistical Analysis

Experiments were performed in triplicate. Data were analyzed by the SPSS software (v25, IBM) using the ONE-way ANOVA and the Tukey's post-hoc test. Significance level was set at $p<0.05$.

\section{Results and Discussion}

\subsection{Fluorescent Microscope Observations}

Fluorescence microscope images acquired on samples Ti6Al4V and Steel 316L bare, coated and after different steps of soaking in water are reported in Figure 1.

The samples without the coating did not fluoresce as expected, while both Ti6Al4V and steel 316L exhibited a fluorescence emission after coating. The fluorescence was almost uniform with some brighter spots probably due to the higher fluorescence of some biomolecules. The coating resulted still present after $3 \mathrm{~h}, 7 \mathrm{~h}, 3$ days and 7 days of soaking in water.

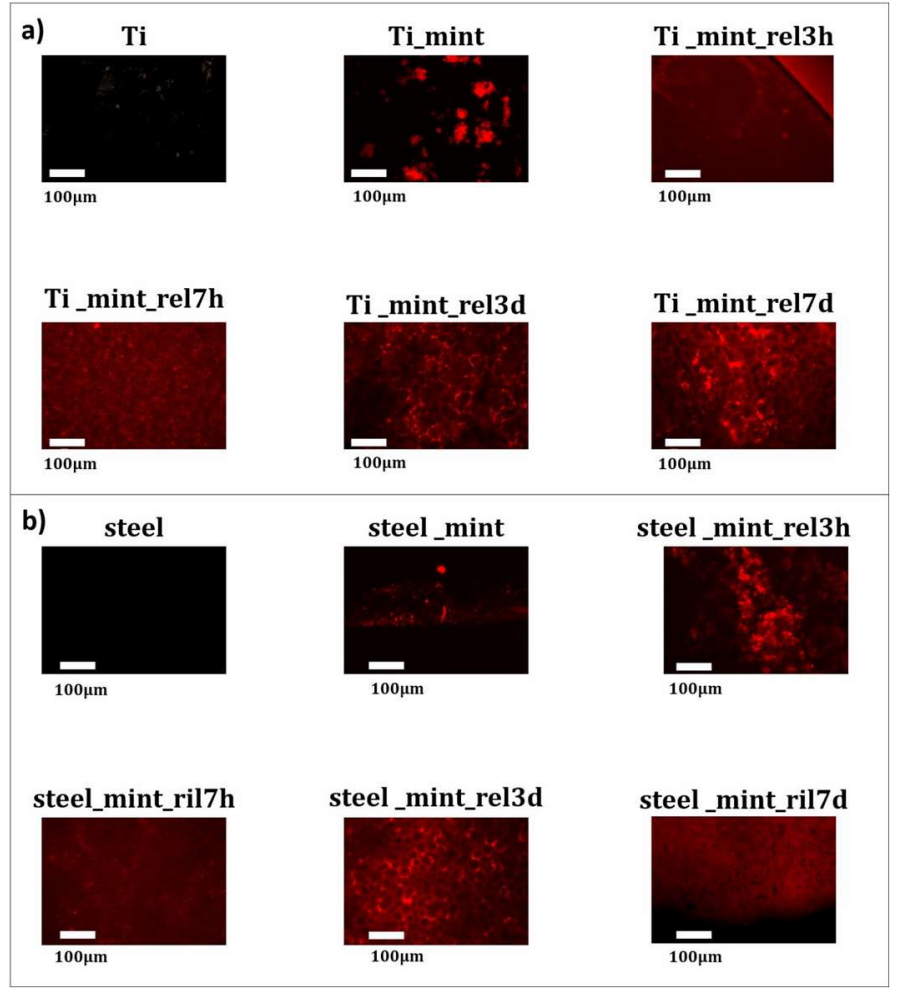

Figure 1. Fluorescent microscope observations (magnification 10×) on samples (a) Ti6Al4V and (b) steel 360L.

\subsection{Tape Tests}

The tape test was performed on all the coated samples before and after soaking in water. The classification resulted as $2 \mathrm{~B}$ (the coating has flaked along the edges and on parts of the squares; the area affected isless than $35 \%$ of the lattice) [12] with partial removing of the outermost layers of the coating for both samples (Steel 316L and Ti6Al4V polished up to 2500). Instead, the steel samples 
polished up to 400 has a classification of 3B (small flakes of the coating are detached along edges and at intersections of cuts; the area affected is less than 15\%-microscope observations reported in Figure 2).

The higher adhesion of the coating to the wrinkled substrate highlights that the increased roughness of the surface positively influences the adhesion of the coating by means of mechanical interlocking.

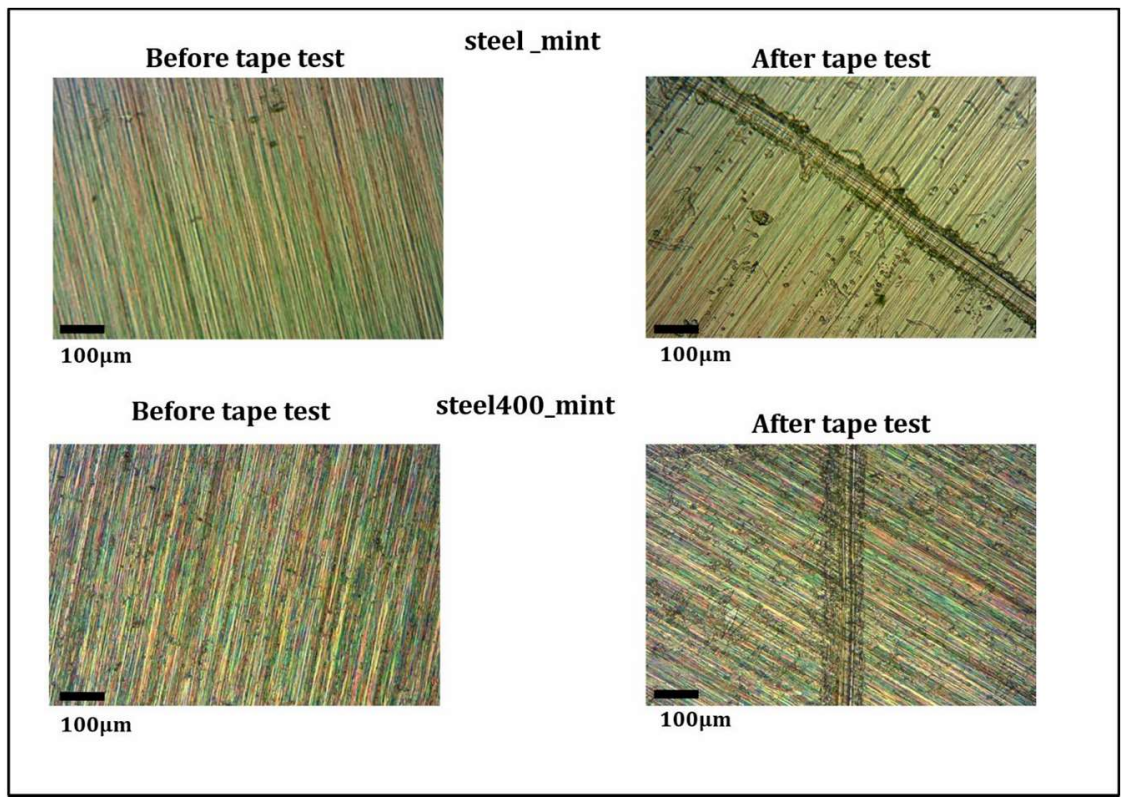

Figure 2. Comparison between smooth steel samples and wrinkled steel samples coated with mint oil before and after the tape tests (magnification 10×).

\subsection{Washing Resistance}

For the steel samples, resistance to acid and basic solutions was also evaluated.

The coating resulted still present after washing with the different solutions as demonstrated by theFTIR analysis reported in Figure 3.

The spectra of all the samples are characterized by the typical vibrational peaks of oxygenated monoterpenes, which are the main components of the Mentha oil. The main peaks are listed in Table 1.

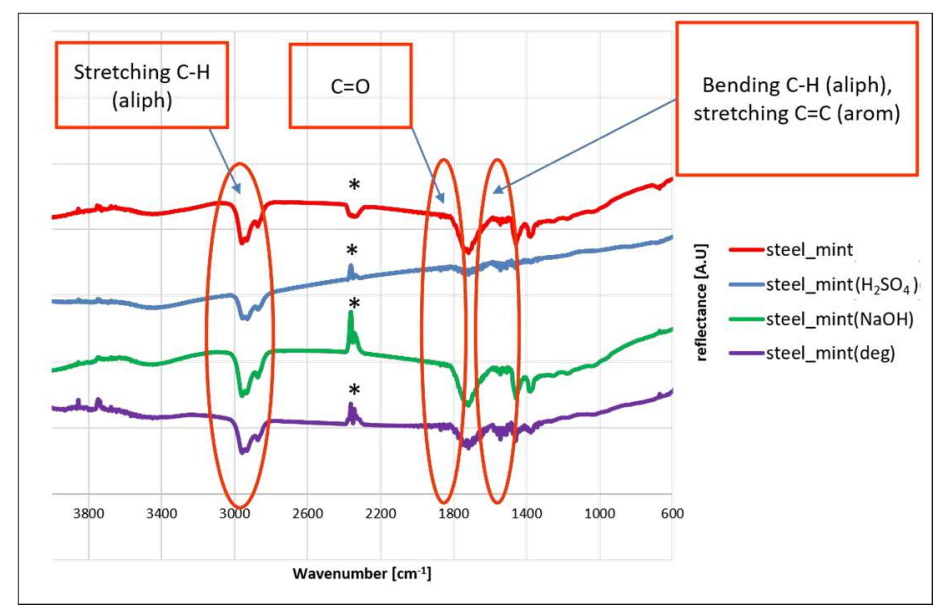

Figure 3. FTIR spectra of the steel samples, coated with mint oil, between 600 and $4000 \mathrm{~cm}^{-1}$ before and after washing with basic and acid solutions. The asterisk * marks the typical band of atmospheric $\mathrm{CO}_{2}$. 
Table 1. Peaks assignments of FTIR spectra.

\begin{tabular}{cc}
\hline Peaks & Assigmements [15] \\
$3450-3200 \mathrm{~cm}^{-1}$ & stretching $-\mathrm{OH}$ \\
$2960-2870 \mathrm{~cm}^{-1}$ & stretching C-H \\
$1780-1700 \mathrm{~cm}^{-1}$ & stretching C=O \\
$1450-1370 \mathrm{~cm}^{-1}$ & bending C-H (aliph), stretching C=C (arom) \\
\hline
\end{tabular}

Only the samples steel_mint $\left(\mathrm{H}_{2} \mathrm{SO}_{4}\right)$ showed a reduced intensity of the bands between 1750 and $1300 \mathrm{~cm}^{-1}$ highlighting the loss of some compounds from the coating and its sensitivity to acid solutions. When washed with basic solutions, which are the main part of the commercial cleaner, the coating remained stable.

\subsection{XPS Analysis on Functionalized Samples}

The success of the procedure of functionalization on CT_50\% mint (funz) samples was checked by means of XPS analysis, because only a very weak signal was detected by FTIR analysis.

The high resolution spectra of carbon and oxygen regions are reported in Figure 4.

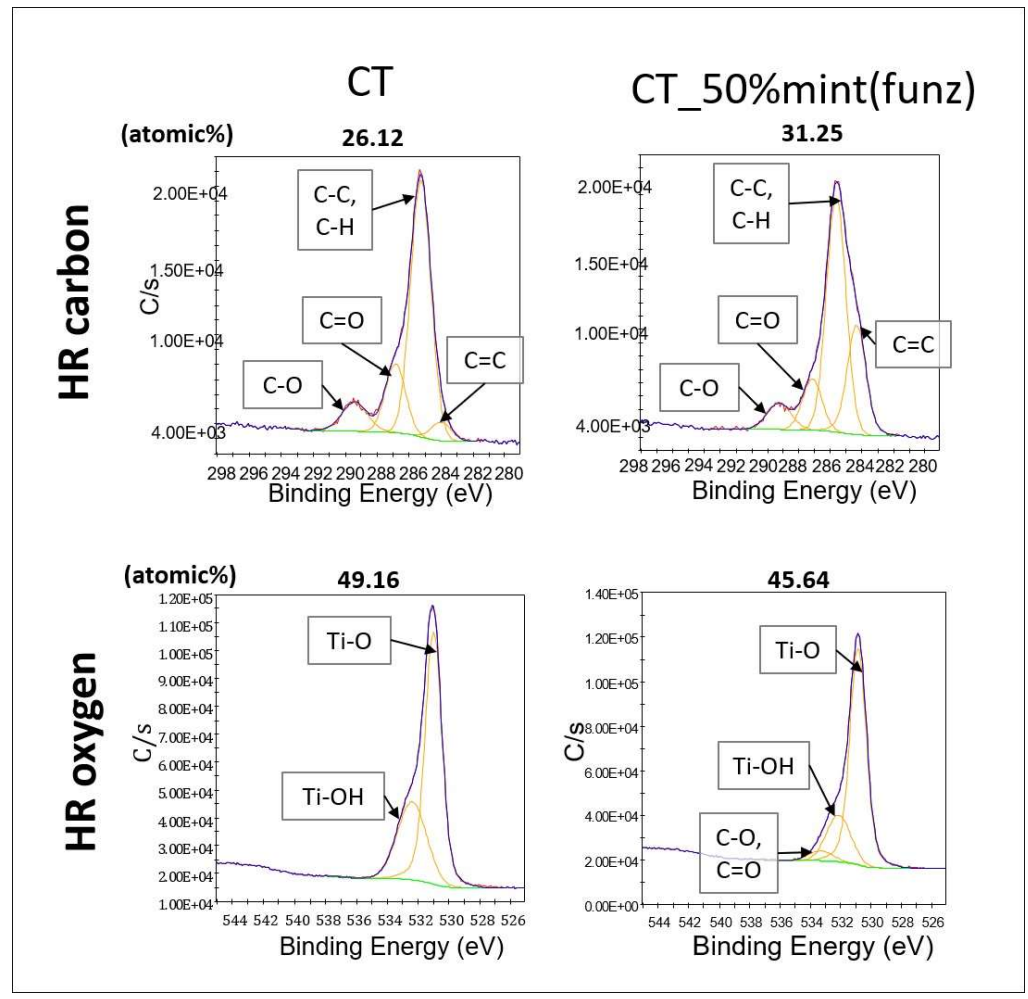

Figure 4. HR spectra of oxygen and carbon regions of samples CT bare and functionalized.

The samples showed an increase in the carbon content after functionalization and also a change of the intensity of the peak at $284 \mathrm{eV}$ of $\mathrm{C}=\mathrm{C}$ bonds, due to the biomolecules of the mint oil bonded to the surfaces. The other peaks at $285.2,286.9$ and $289.12 \mathrm{eV}$ correlated respectively with $\mathrm{C}-\mathrm{C}$ and $\mathrm{C}-\mathrm{H}$ bonds, $\mathrm{C}-\mathrm{O}$ bonds and $\mathrm{C}=\mathrm{O}$ bonds are correlated with some biomolecules of the mint oil, but could also be ascribed to the surface contaminants that are affine to titanium surface considering that they do not significantly change after functionalization $[8,9,16]$.

The HR spectra of the oxygen region showed for the CT samples the presence of two peaks due to the Ti-O bonds of titanium oxide $(530 \mathrm{eV})$ and to the $\mathrm{Ti}-\mathrm{OH}(532 \mathrm{eV})$ bonds due to the chemical treatment. On the spectrum of the functionalized samples, it is possible to see a reduction in the intensity of the peak due to $\mathrm{Ti}-\mathrm{OH}$ bonds and the presence of a peak at $533.9 \mathrm{eV}$ due to the presence of $\mathrm{C}-\mathrm{O}$ and $\mathrm{C}=\mathrm{O}$ bonds highlighting that some biomolecules from the mint oil are bonded to the $\mathrm{OH}$ groups on the surface $[8,9,17]$. 
It can be concluded that both coating and functionalization allow to cover metal surfaces with mint oil, but, according to the different procedures, different types of biomolecules are grafted, in different amount and requiring different techniques of surface analysis.

\subsection{Antibacterial Tests}

Results obtained from alamar blue analysis on the samples surfaces are reported in Figure 5.

The mint coating was able to reduce the amount of adherent metabolically active bacteria in a significant manner in comparison with bare control materials after 24 and $48 \mathrm{~h}$ of incubation. However, when the same results were considered in function of time, both control and mint coated samples showed an increasein terms of viable colonies, even if the difference between the two groups were still significant. The data highlighted a bacteriostatic effect of the coating.

In general, none inhibitory effect was observed in the collected water specimens (data reported in Table 2). Accordingly, the mint coating seems not to release antibacterial compounds, which could induce cytotoxicity, but it acts through a contact mechanism.

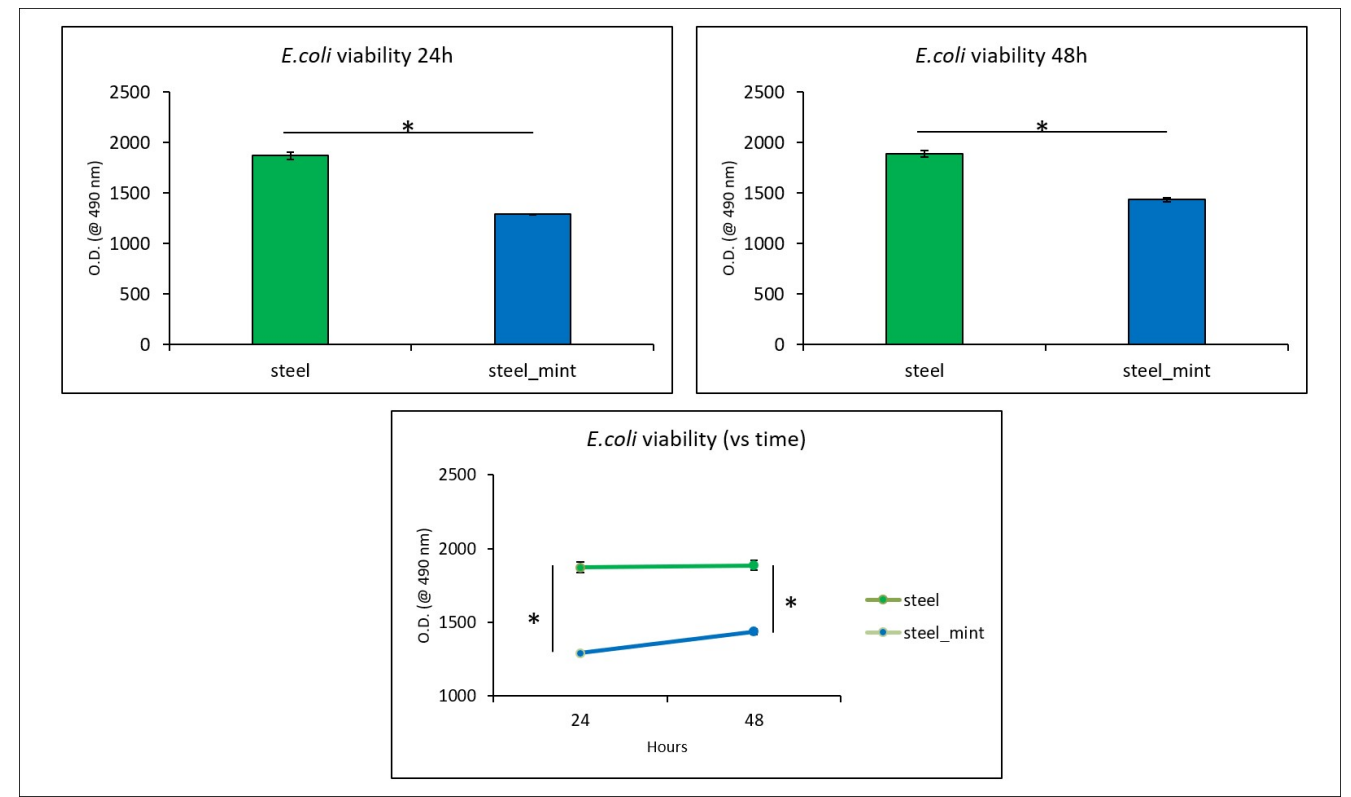

Figure 5. Alamar blue assay results after $24 \mathrm{~h}, 48 \mathrm{~h}$ and in function of time. Bars represent meansand standard deviations; ${ }^{*}=p<0.05$.

Table 2. Water optical density (o.d.) after 24 and $48 \mathrm{~h}$ in direct contact with bare and mint doped samples.

\begin{tabular}{ccc}
\hline Water from Sample & o.d.@24 h (means \pm dev.st) & o.d.@48 h (means \pm dev.st) \\
\hline steel & $0.035( \pm 0.0035)$ & $0.046( \pm 0.0017)$ \\
steel_mint & $0.035( \pm 0.0040)$ & $0.041( \pm 0.0023)$ \\
\hline
\end{tabular}

\section{Conclusions}

The relevance of the topic of this work deals with the need for innovation in the development of anti-adhesive surfaces, since a range of medical, laboratory and general purpose instrumentation requires antibacterial safety.

To this aim, stainless steel $316 \mathrm{~L}$ and Ti6Al4V, which are alloys used in the fabrications of different devices, were coated with Mentha piperita essential oils.

The procedure of coating was developed for non-implantable device, while functionalization of the surface of chemically treated titanium alloy (CT) was developed for implant applications in contact with bone.

The success of functionalization and coating treatments was highlighted by XPS or FTIR analysis that showed the presence of different biomolecules of the mint oil on the surface of the samples, according to the different procedures. 
The coating on both stainless steel and Ti6Al4V samples resulted stable also after 7 days of soaking in water, as observed by fluorescence microscopy. Moreover, the tape test performed on steel samples showed agood stability of the coating which was increased by a higher roughness of the surfaces before the coating procedure.

The coated steel samples were also washed with acid and basic solutions in order to test the resistance to cleaning and analyzed by means of FTIR spectroscopy which showed that the coating resists to basic cleaner and has only a little damage after acid cleaning.

The mint coating on steel showed the ability to reduce the E. coli surface contamination highlighting an anti-adhesive behavior.

The procedure of coating and functionalization performed with an essential oil seems to be a promising strategy to exploit their antibacterial activity for both non-implantable and implantable applications in biomedical field and it can be easily extended to other essential oils.

Author Contributions: Conceptualization, M.C., S.F., V.A., G.B. and S.S.; Methodology, M.C., S.F. and S.S.; Formal Analysis, A.C., L.R., G.G. and C.N. ; Investigation, M.C., S.F., G.B., C.B., V.A., C.N., G.G., A.C. and L.R.; Resources, G.B., S.S. and L.R.; Data Curation, M.C., G.B., V.A., A.C., C.N. and C.B..; Writing-Original Draft Preparation, M.C., S.F., S.S.; Writing-Review and Editing, G.B., V.A., C.B., G.G., C.N., A.C. and L.R.; Visualization, X.X.; Supervision, S.S., G.B., and L.R.

Funding: This work was in part supported by a research grant from Fondazione Cassa di Risparmio diTorino $(\mathrm{RF}=2015.1681)$.

Acknowledgments: We thank Thermo Fisher Scientific for the assistance with the XPS analysis and Professor FrancescoGeobaldo for his help in FTIR results interpretation. We also thank Professor Alessandro Bistolfi for his contribution to the project.

Conflicts of Interest: The authors declare no conflict of interest.

\section{References}

1. El Asbahani, A.; Miladi, K.; Badri, W.; Sala, M.; Addi, E.A.; Casabianca, H.; El Mousadik, A.; Hartmann, D.; Jilale, A.; Renaud, F.N.R.; et al. Essential oils: From extraction to encapsulation. Int. J. Pharm. 2015, 483, 220243.

2. Grassmann, J.; Elstner, E.F. Essential oils. Properties and usage. In Encyclopedia of Food Sciences and Nutrition; Caballero, B., Ed.; Academic Press: Cambridge, MA, USA, 2003; pp. 2177-2184.

3. Raut, J.S.; Karuppayil, S.M. A status review on the medicinal properties of essential oils. Ind. Crops Prod. 2014, 62, 250-264.

4. Di Pasqua, R.; Betts, G.; Hoskins, N.; Edwards, M.; Ercolini, D.; Mauriello, G. Membrane Toxicity of Antimicrobial Compounds from Essential Oils. J. Agric. Food Chem. 2007, 55, 4863-4870.

5. Yap, P.S.X.; Yiap, B.C.; Ping, H.C.; Lim, S.H.E. Essential oils, a new horizon in combating bacterial antibiotic resistance. Open Microbiol. J. 2014, 8, 6-14.

6. Nagar, G.B.; Pradesh, U. In vitro antimicrobial activity, phytochemical analysis and total phenolic content of essential oil from Mentha spicata and Mentha piperita. Int. Food Res. J. 2015, 22, 2440-2445.

7. Kamatou, G.P.P.; Vermaak, I.; Viljoen, A.M.; Lawrence, B.M.; Menthol: A simple monoterpene with remarkable biological properties. Phytochemistry 2013, 96, 15-25.

8. Ferraris, E.V.S.; Spriano, S.; Bianchi, C.L.; Cassinelli, C.; Surface modification of Ti-6Al-4V alloy for biomineralization and specific biological response: Part II, alkaline phosphatase grafting. J. Mater. Sci. Mater. Med. 2011, 22, 1835-1842.

9. Ferraris, S., Spriano, S., Pan, G., Venturello, A., Bianchi, C.L., Chiesa, R., Faga, M.G., Maina, G.; Verne, E. Surface modification of Ti-6Al-4V alloy for biomineralization and specific biological response: Part I, inorganic modification. J. Mater. Sci. Mater. Med. 2011, 22, 533-545.

10. Cazzola, M., Ferraris, S., Boschetto, F., Rondinella, A., Marin, E., Zhu, W., Pezzotti, G., Vernè, E.; Spriano, S., Green tea polyphenols coupled with a bioactive titanium alloy surface: In vitro characterization of osteoinductive behavior through a KUSA A1 cell study. Int. J. Mol. Sci. 2018, 19, 2255.

11. Alankar, S. A review on peppermint oil. Asian J. Pharm. Clin. Res. 2009, 2, 27-33.

12. ASTM D3359-17 Standard Test Methods for Rating Adhesion by Tape Test; ASTM International: West Conshohocken, PA, USA, 2017. 
13. Ferraris, M.; Perero, S.; Ferraris, S.; Miola, M.; Vernè, E.; Skoglund, S.; Blomberg, E.; Wallinder, I.O. Applied Surface Science Antibacterial silver nanocluster/silica composite coatings on stainless steel. Appl. Surf. Sci. 2017, 396, 1546-1555.

14. Hevesi, K.; Scarso, F. Substrate with Antimicrobial Properties. Eur. Patent WO/2007/147832, 27 December 2007.

15. Prakash, N.; Yumus, M. fourier transform infrared spectroscopy analysis of oilof menthaarvensis grown at sites varying with vehicular traffic loads in Lucknow city, India. Int. J. Environ. 2013, 2, 16-25.

16. Thermo Scientific. XPS Simplified: Carbon. Avaliable online: http://www.xpssimplified.com/elements/carbon.php (accessed on 15 November 2017).

17. Thermo Scientific. XPS Simplified: Oxygen. Avaliable online: http://xpssimplified.com/elements/oxygen.php (accessed on 7 December 2017).

(C) 2019 by the authors. Licensee MDPI, Basel, Switzerland. This article is an open access article distributed under the terms and conditions of the Creative Commons Attribution (CC BY) license (http://creativecommons.org/licenses/by/4.0/). 